2. Hensgens MP, Dekkers OM, Demeulemeester A, et al. Diarrhoea in general practice: when should a Clostridium difficile infection be considered? Results of a nested case-control study. Clin Microbiol Infect 2014;20:01067-O1074.
3. Dubberke ER, Han Z, Bobo L, et al. Impact of clinical symptoms on interpretation of diagnostic assays for Clostridium difficile infections. J Clin Microbiol 2011;49:2887-93.

DOI: 10.1056/NEJMc1505190

\title{
Compliance with Results Reporting at ClinicalTrials.gov
}

TO THE EDITOR: The low compliance with ClinicalTrials.gov reporting requirements that Anderson et al. (March 12 issue) ${ }^{1}$ documented among clinical research sponsors should garner attention. As the research enterprise addresses this serious ethical breach, however, we must remember an equally binding obligation to share research findings with the clinical trial participants who make scientific advances possible. ${ }^{2}$

Most participants (77\%) are never informed of the trial's results, and $72 \%$ say that it is "very important" to address this issue. ${ }^{3}$ In a new report, the Institute of Medicine calls the sharing of results in understandable language a "matter of public transparency and respect" and cites research by the authors of this letter demonstrating feasibility. ${ }^{4}$ The Multi-Regional Clinical Trials Center at Harvard developed a comprehensive guidance document and toolkit to deconstruct and ease the process. ${ }^{5}$

Although ClinicalTrials.gov currently does not address this unethical situation, it could - the Food and Drug Administration (FDA) Amendments Act of 2007 authorized the Department of Health and Human Services to require technical and narrative results summaries. We hope the work by Anderson et al. will serve as a call to improve registration and reporting not only for the scientific community but also for patients and the public. It is our obligation, and it is the right thing to do.

Zachary P. Hallinan, B.A.

Kenneth A. Getz, M.B.A.

Center for Information and Study on Clinical Research Participation Boston, MA

zhallinan@ciscrp.org

Barbara E. Bierer, M.D.

Multi-Regional Clinical Trials Center at Harvard University Boston, MA

No potential conflict of interest relevant to this letter was reported.

1. Anderson ML, Chiswell K, Peterson ED, Tasneem A, Topping J, Califf RM. Compliance with results reporting at ClinicalTrials.gov. N Engl J Med 2015;372:1031-9.
2. World Medical Association. World Medical Association Declaration of Helsinki: ethical principles for medical research involving human subjects. JAMA 2013;310:2191-4.

3. Kost RG, Lee LM, Yessis J, Wesley RA, Henderson DK, Coller BS. Assessing participant-centered outcomes to improve clinical research. N Engl J Med 2013;369:2179-81.

4. The clinical trial life cycle and when to share data. In: Sharing clinical trial data: maximizing benefits, minimizing risk. Washington, DC: National Academies Press, 2015:91-138.

5. Multi-Regional Clinical Trials Center at Harvard University return of results guidance document (http://mrct.globalhealth .harvard.edu/file/341171).

DOI: 10.1056/NEJMc1504513

THE AUthors Reply: Hallinan et al. underscore the ethical duty of research sponsors to share findings with trial participants and suggest that providing nontechnical and technical narrative summaries could help fulfill this obligation. Notably, the notice of proposed rulemaking for the FDA Amendments Act ${ }^{1}$ has delayed enactment of these summaries, pending public feedback.

Additional issues are presented when sponsors delay reporting while pursuing marketing approval for novel products. We estimated that 44 to $45 \%$ of industry-funded trials (as compared with $6 \%$ of trials funded by the National Institutes of Health and $9 \%$ of other trials) were not required to report because they involved an unapproved or unlabeled product. Under proposed rules, sponsors seeking approval for new products or indications may delay reporting to ClinicalTrials .gov for 2 more years if a certification of delay is filed before the 1-year deadline. Trials involving unapproved products that do not include plans for seeking approval must report within 1 year. Although this rule ensures eventual public access to data, it also highlights tensions between desires to protect trade secrets and obligations to share knowledge. Furthermore, delays in public reporting may lead to unnecessarily duplicative trials and expose participants to unwarranted risks.

Monique L. Anderson, M.D.

Duke University School of Medicine

Durham, NC

monique.anderson@duke.edu 
Eric D. Peterson, M.D., M.P.H.

Duke Clinical Research Institute

Durham, NC

Since publication of their article, the authors report no further potential conflict of interest.
1. Department of Health and Human Services. Notice of proposed rulemaking: clinical trials registration and results submission. Fed Regist 2014;79(225):69566-680.

\section{Trial-Results Reporting and Academic Medical Centers}

TO THE EDITOR: Reporting of aggregate results helps mitigate disclosure biases affecting medical research. ${ }^{1}$ Although the reporting of summary results is currently mandated by the Food and Drug Administration Amendments Act of 2007 (FDAAA), ${ }^{2}$ published findings suggest underreporting. ${ }^{3}$ Two recent proposals are aimed at improving public reporting of aggregate results.
These are a Notice of Proposed Rulemaking $(\mathrm{NPRM})^{4}$ to expand FDAAA requirements to include the results of trials of unapproved products, and a draft policy requiring the results of all National Institutes of Health (NIH)-funded trials, including those not subject to the FDAAA. ${ }^{5}$ Noncompliance with either requirement would carry a risk of NIH-funding loss and other finan-

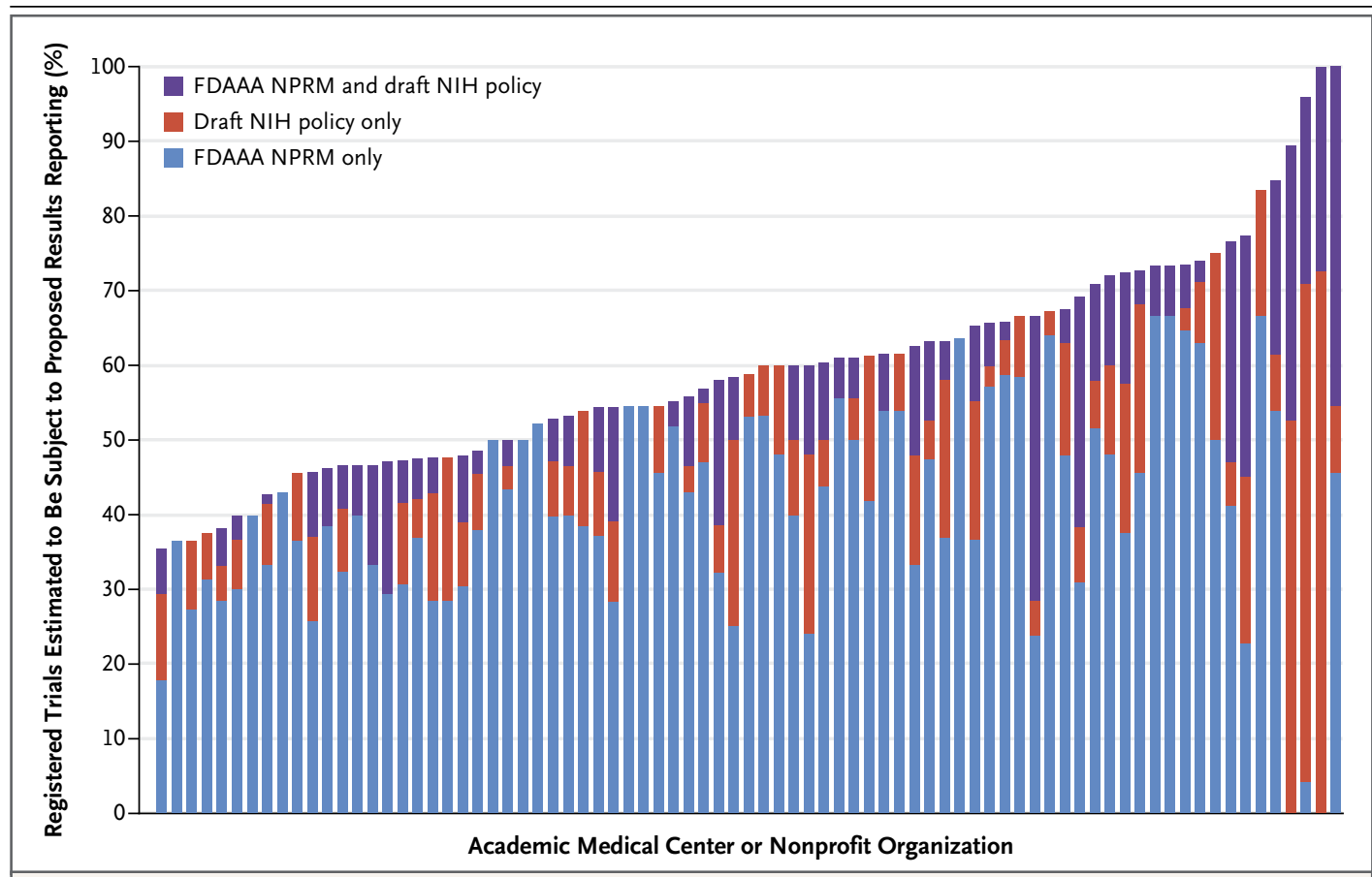

Figure 1. Trials by 80 Sponsors Estimated to Be Subject to Proposed Results Reporting.

Each bar represents an academic medical center or other nonprofit organization with 10 or more registered trials in the sample, arranged according to the total percentage of registered trials sponsored by that organization and estimated to be subject to at least one of the two proposed results-reporting requirements. Algorithms involving ClinicalTrials.gov data elements (http://prsinfo.clinicaltrials.gov/definitions.html) were used to estimate trials subject to each proposal. For the Food and Drug Administration Amendments Act of 2007 (FDAAA) Notice of Proposed Rulemaking (NPRM), the algorithm and data elements were an "Interventional" Study Type; a Study Phase other than "0" or "1," at least one Intervention Type as "Drug," "Biologic" (or "Genetic"), or "Device" (or "Radiation"); at least one Facility Country as "United States" or Investigational New Drug Application (IND) or Investigational Device Exemption (IDE) Status as "Yes" (not available publicly); and a Primary Completion Date (if missing, Study Completion Date) as "January 2008" or later. For the draft National Institutes of Health (NIH) policy, the algorithm and data elements were an "Interventional" Study Type and at least one NIH Institute or Center as Collaborator. 